\section{Academics can help shape Wikipedia}

Public understanding of science is increasingly important. Wikipedia is widely used by students, educators, researchers, doctors, journalists, and policy-makers. The online, crowd-sourced encyclopedia site is perceived as increasingly trustworthy, making it a key public engagement platform with immediate impacts on scientific literacy (1).

Now is an important time in the evolution of the encyclopedia. Its parent organization, the Wikimedia Foundation, is working to shape its strategic focus through to 2030. This represents an unprecedented opportunity for the global scientific community to advise on its future. Wikipedia has discussion pages for users to provide feedback on some of the upcoming challenges (2).

The scientific community can improve Wikipedia on a more granular level by learning to edit the encyclopedia in areas that need improvement. Poorly written articles can mislead readers and give a false impression of a research field. The recent introduction of a new editing interface has made the encyclopedia as easy to edit as a Word document, and a short 2014 article outlines some editing advice for scientists (3).

Wikipedia is increasingly engaging expert communities to improve accuracy and coverage. Interested parties can contribute to several existing collaborative initiatives, or propose new ones. For example, some academic journals (such as PLOS Computational Biology, Gene, and WikiJournal of Medicine) have agreed to dual-publish articles as both a citable publication and Wikipedia page (4). The Cochrane library, a collection of healthcare databases, has a similar quality-improvement partnership to help integrate optimal scientific references into the encyclopedia (5).

Finally, the new Wikidata system stores machine-readable, structured data, complementary to the prose format of the encyclopedia. Integrating Wikidata with scientific databases provides new opportunities to discover, curate, and use scientific knowledge within and across domains (6).

Thomas Shafee, ${ }^{*}$ Daniel Mietchen, ${ }^{2}$ Andrew I. Su ${ }^{3}$

1'La Trobe University, Melbourne, VIC 3086, Australia. 2University of Virginia, Charlottesville, VA 22903, USA ${ }^{3}$ The Scripps Research Institute, La Jolla, CA 92037, USA.

*Corresponding author.

Email: T.Shafee@LaTrobe.edu.au
REFERENCES

1. D. Jemielniak, E. Aibar, J. Assoc. Inform. Sci. Technol. 67, 1773 (2016).

2. Wikimedia movement, Strategy 2030 (https://meta.wikimedia.org/wiki/Strategy/Wikim edia_movement/2017/Participate).

3. D. W. Logan, M. Sandal, P. P. Gardner, M. Manske, A. Bateman, PLOS Comput. Biol. 6, 10.1371/journal.pcbi.1000941 (2010).

4. T. Shafee, J. Heilman, G. Masukume, M. Häggström, "Wikipedia's medical content: A new era of collaboration," WikiMedia Foundation Blog (2016).

5. S. de Haan, "Wikipedia: An important dissemination tool for Cochrane," Cochrane Community (2017); http://community.cochrane.org/news/wikipediaimportant-dissemination-tool-cochrane.

6. S. Burgstaller-Muehlbacher et al., Database 2016, 10.1093/database/baw015 (2016).

DOI:10.1126/science.aao0462

URL:http://science.sciencemag.org/content/357/6351/ $\underline{557.2}$ 